\title{
A computer-controlled vapor-dilution olfactometer
}

\author{
VERNON A. BENIGNUS and JAMES D. PRAH \\ U.S. Environmental Protection Agency, Chapel Hill, North Carolina 27514 and \\ University of North Carolina, Chapel Hill, North Carolina 27514
}

\begin{abstract}
A computer-controlled olfactometer with several innovations leading to closer control over the stimulus parameters and minimization of nonolfactory cues is presented. A microcomputer is used to control the stimulus duration and interstimulus interval. Electronic mass flow controllers are employed to maintain close control over the flow in the pure air line and the stimulus-bearing line. Both humidity and temperature are closely regulated. Using a nasal catheter, this stimulus delivery system coupled with velopharyngeal closure and oral breathing permits accurate specification of the quantity of stimulus material actually entering a subject's nares. The design is such that the computer programming, patch panel interface, and threaded tubing allow versatility in the type of olfactometric study that can be conducted. This system is amenable for threshold, electrophysiological, and sensory adaptation studies of olfaction.
\end{abstract}

Numerous techniques for the delivery of odorant materials to human subjects have been developed. These techniques have included static dilution (Teghtsoonian, Teghtsoonian, Berglund, \& Berglund, 1978), vapor diffusion (Nader, 1958), continuous vapor dilution (Cain, 1974), and microencapsulation (Davis, 1979). These techniques have been applied to devices varying in size from portable (Lindvall, 1970) to room-sized (Schneider \& Wolf, 1955). Since there is so much variety in construction and delivery techniques, the reader is referred to Dravnieks (1975), Johnston (1967), and Mateson (1955) for reviews.

The olfactometer discussed here offers some unique solutions to the problems of stimulus control and delivery. Stone, Pryor, and Steinmetz (1969) have suggested some criteria for olfactometric design that are relevant: (1) continuous flow of diluent air, (2) flexibility in selection of stimulus and stimulus concentration, (3) rapid introduction and removal of the stimulus, and (4) ease of operation, data recording, and apparatus cleaning. Additional requirements that appear germane are: (5) cue avoidance, (6) temperature and humidity control, (7) airstream calibration, and (8) objective evaluation of the odorant concentrations. The olfactometric system to be described partially meets these requirements through computer control and the use of electronic mass flow controllers (EMFC).

\section{DESIGN CHARACTERISTICS}

Figure 1 illustrates the basic design. The apparatus is housed in a modified Thelco oven (Model 28). Modifi-

Reprint requests should be sent to James D. Prah, U.S. Environmental Protection Agency, Neurotoxicology Division, Medical Research Building C, No. 224H, Mason Farm Road, University of North Carolina, Chapel Hill, North Carolina 27514. cations of the temperature regulation circuit (Figure 2) reduce the temperature variation to $\pm .15^{\circ} \mathrm{C}$. Closely regulated temperature is necessary to avoid variations in the vapor pressure of the odorant, which is strongly temperature dependent. Failure to control this temperature could yield significant variations in stimulus strength.

The air used for the diluent and pure air lines is "zero" air, which has less than 1 ppm of hydrocarbons. After entering the olfactometer, the air is filtered with a Pyrex glass wool/silica gel (SG)/active charcoal (AC)/ Pyrex glass wool filter. The glass wool input filter removes particulates that may remain in the airstream, and the glass wool output filter removes those that may be produced by the SG or $\mathrm{AC}$ elements of the filter itself. The SG filter serves as a dessicant and as an adsorbent of polar compounds, and the AC filter favors the adsorption of apolar compounds (Weurman, 1974). Additionally, there is some evidence that microorganismic growth (Foster, Scofield, \& Dallenbach, 1950) can take place on AC. For this reason, the airstream is dessicated first by SG. An additional AC filter is used after the EMFCs (Tylan FM260 and Matheson Model 8240) due to the inclusion in them of a small butyl rubber O-ring. In the case of the $0-$ to $300-\mathrm{ml}$ EMFC, the AC filter is used after the EMFC instead of both before and after. The use of EMFC allows maintenance of $\pm .25 \%$ of the set flow value. The EMFCs continuously compensate for changes in flow, thus maintaining constant flow.

With the exception of the butyl rubber O-ring in the EMFCs, all parts that contact the airstream are glass, 316 stainless steel (SS), or Teflon. These parts are all thoroughly cleaned first with chromic acid and then thoroughly rinsed with deionized water. The water used to humidify the airstream is deionized.

The three-way solenoid valves used in this assembly 


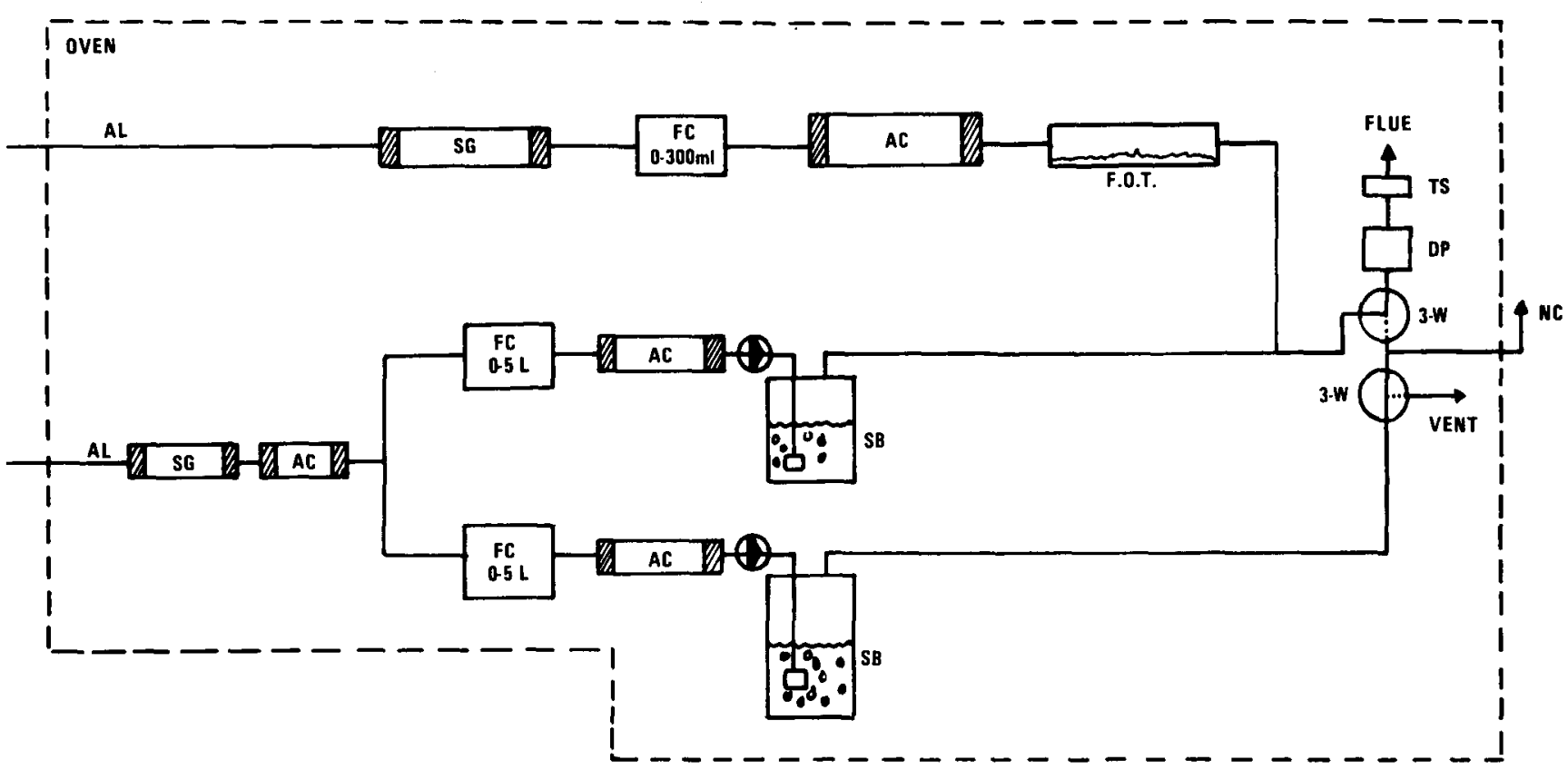

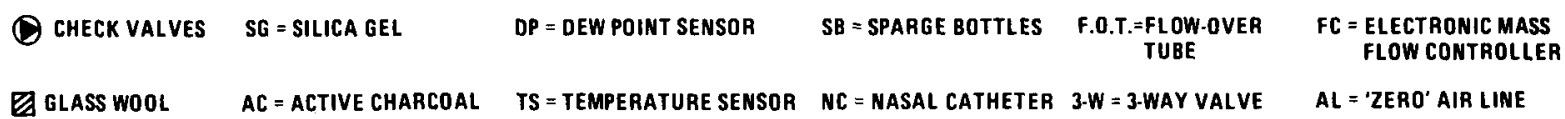

Figure 1. Schematic of olfactometer (not to scale).

are made by General Valve (Model 1-29-900). All contacting parts of these and the check valves (Laboratory Supply Company, Model G) are Teflon. The humidity level is monitored via a dew-point sensor (General Eastern 1100AP), and the temperature is monitored by its associated temperature sensor.

All of the various connecting tubing is of $1 / 8$-in. (3.2-mm) or $1 / 4-$ in. $(6-\mathrm{mm})$ Teflon or $316 \mathrm{SS}$. The 3.2-mm Teflon tubing is fitted with $1 / 4-28$ Altex
(Rainin Model 200-10) fittings that allow easy and rapid assembly and disassembly for modification or cleaning. The output to the subject consists of a $6-\mathrm{mm}$ 316 SS tube rigidly attached to a styrofoam lined metal chassis box $(25.5 \times 9 \times 15 \mathrm{~cm})$. The tube is firmly bolted to avoid movement upon the switching of the stimulus and pure air lines. The lined metal box permits only the Swagelok fitting to which the nasal catheter attaches to be exposed to the ambient air. This reduces

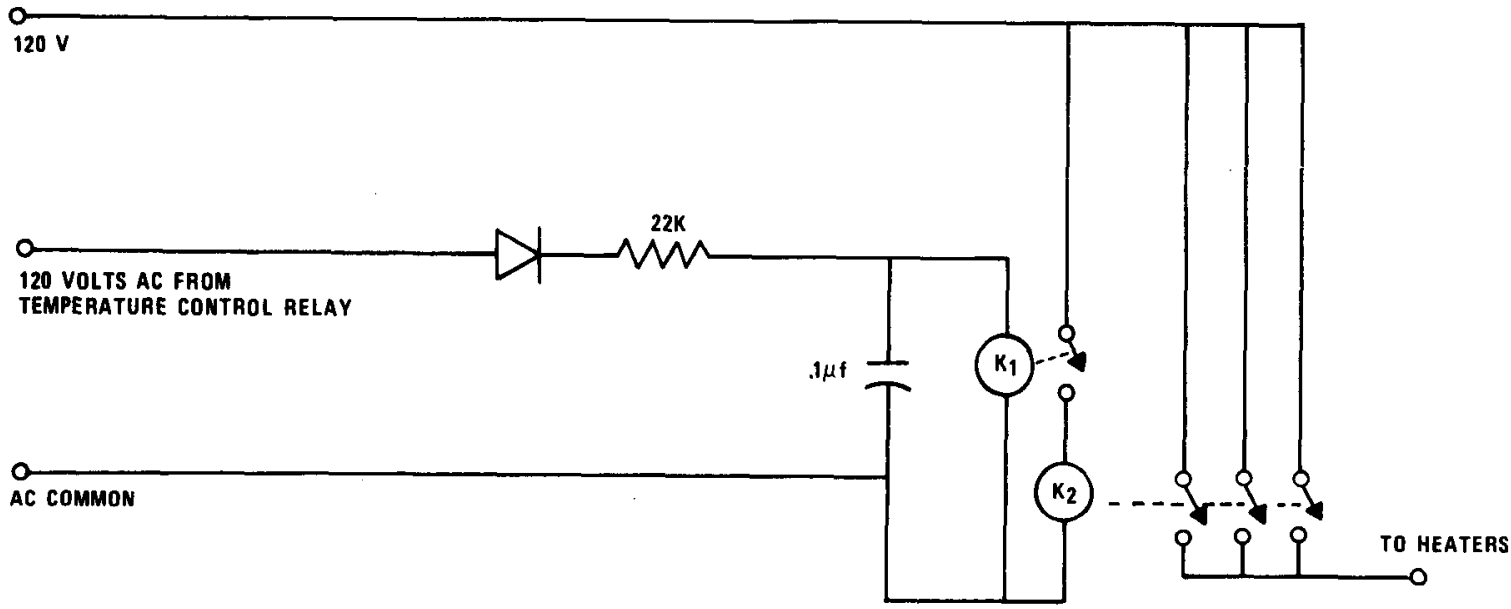

Figure 2. This circuit bypasses the normal oven thermostatic control and substitutes a low-hysteresis mercury-controlled thermostat (Versa Therm Model 2149). This reduces the temperature variation to $\pm .15^{\circ} \mathrm{C}$. The low-power relay (K1) is controlled by the thermostat and, when closed, activates the high-current (K2), which supplies current to the oven heaters. The diode requirements are $<200$ PIV and $500-\mathrm{mA}$ forward current. 
heat loss and condensation in the tube. The nasal catheters were constructed by filing 6-mm 316 SS female fittings to be easily and comfortably inserted into the subject's naris while ensuring an airtight fit. The height of the nose piece is constant $(1.2 \mathrm{~cm})$, but the width at the base ranges from 1.4 to $1.8 \mathrm{~cm}$. The width at the top varies from .8 to $1.2 \mathrm{~cm}$.

A Dynabyte Basic Controller (DBC) with $16 \mathrm{~K}$ bytes of RAM and $4 \mathrm{~K}$ bytes of EPROM is interfaced with the olfactometer through a patch panel. This permits the connection of the various memory-mapped DBC relays, flag outputs, or sense inputs to the olfactometer and allows the rapid modification of the olfactometric function and timing parameters via the patch panel and programming.

\section{OPERATION}

The airstream enters the olfactometer and is filtered, and the flow level is set by the EMFCs. The pure air line and the line used as diluent are humidified by flowing the air through 1-liter sparge bottles nearly filled with deionized water. The odor line enters the flowover tube $(14 \times 2 \mathrm{~cm})$ in which the head space is saturated and emerges as an odorant-saturated flow. A flowover system was used to avoid aerosol generation in a sparge bottle. Varying this flow and the diluent airflow varies the odorant concentration. The stimulus-bearing air passes through the three way switching valve to the dew point and temperature sensors and out the flue. The nonodorized air flows to the subject until the three-way valves are actuated, at which time the stimulus and the neutral air lines are switched for the desired stimulus duration.

The subjects are trained to breathe slowly and gently through the mouth while maintaining velopharyngeal closure. With this technique, the continuous pure airflow enters one naris and exits the other. This is similar to the technique suggested by Mozell (1971) using the Valsalva maneuver and an olfactometer input to one naris and a vacuum at the other to establish the complete olfactory stimulation, a sort of artificial sniff. Stimulation is obtained by substituting the stimulus air line for the neutral air line. With this paradigm, the stimulus flow does not interact with pressure and flow changes that take place during breathing. The stimulus onset does not have to be coordinated with the breathing cycle. While it is not known how much odorant material actually contacts the olfactory mucosa (DeVries \& Stuiver, 1961, estimate 2\%), the quantity actually entering the naris can be specified with this technique.

To avoid olfactory cues, the parts of the apparatus exposed to either the stimulus or pure air lines are cleaned with chromic acid and thoroughly rinsed with deionized water. In an effort to verify the freedom from contamination, an analysis of the particulate level in the purified airstream after the second filter was conducted. A condensation nuclei counter that responds to particles with diameters of $.0025 \times 10^{-6} \mathrm{~m}$ and larger (Environment/One Corporation, Model Rich 100) received direct input from the filter. This instrument, with a minimum resolution of $50 \mathrm{nuclei} / \mathrm{ml}$, indicated a particulate level of 60-150 nuclei $/ \mathrm{ml}$. By comparison, when room air was sampled through a HEPA .2-micrometer filter (Gelman Model 12106) by the monitor, the obtained particle count was $60-160$ nuclei $/ \mathrm{ml}$. Even the cleanest air contains several hundred particles per milliter (Air Quality Critera for Particulate Matter, 1970).

Further, a $.2 \times 10^{-6} \mathrm{~m}$ pore size filter (Millipore FGLP04700) was attached to the output of the olfactometer filter, and after flowing 2 liters/min of purified air for $5 \mathrm{~h}$, no measurable particulate matter had accumulated. A gas chromatographic analysis (Hewlett-Packard $7620 \mathrm{~A}$, flame ionization, $1.83 \mathrm{~m}, 10 \%$ Silar $10 \mathrm{C} 100-1$ 120-mesh column) of the effluent of the filter revealed no detectable contaminant. While these data do entirely not eliminate the possibility of contamination either by particulate matter or by eluted chemicals, they do indicate that if any contamination is present, it is extremely low and is unlikely to have had a negative impact upon the quality of the purified airstream. The gas chromatograph is also used to calibrate the odorant level in the stimulus line and to ensure purity of the odorant.

A constant high relative humidity (RH) $>75 \%$ is preferred, and the output temperature is maintained at body temperature to avoid the possibility of the subject's confusing the dessication or cooling of the nasal mucosa with olfactory simulation. Benignus and Prah (1980) found that the threshold for detection of pure airflow was highest, 2.4 liters $/ \mathrm{min}$, at $>75 \% \mathrm{RH}$ and at body temperature $\left(37^{\circ} \mathrm{C}\right)$. The lowest flow threshold, less than .4 liters $/ \mathrm{min}$, was found at $<5 \%$ RH regardless of the temperature. These results indicate that flow threshold is dependent to a large extent on RH. Since subjects may confuse trigeminally mediated air flow with olfactory sensation, this critical variable should be specified and controlled as a potentially confounding variable in the design of an olfactometer. The practical import of this finding is that one can, if an $\mathrm{RH}>75 \%$ and body temperature stimulus is utilized, use a greater range of subthreshold flow rates. The greater flow range allows easier control over the stimulus, since it is easier to control large flows than small flows.

Initially, the two three-way valves were arranged so that when switched simultaneously they functioned as a four-way valve. When the odorant was switched from the flue to the subject, it was found that a subject-detectable pressure surge resulted. This surge occurred due to an approximately $15-\mathrm{msec}$ delay in switching that resulted in a brief period of no flow and consequent pressure buildup. This problem was resolved by switching the stimulus air line slightly before $(30 \mathrm{msec})$ switching out the neutral line. To ensure that no differential flow rate 


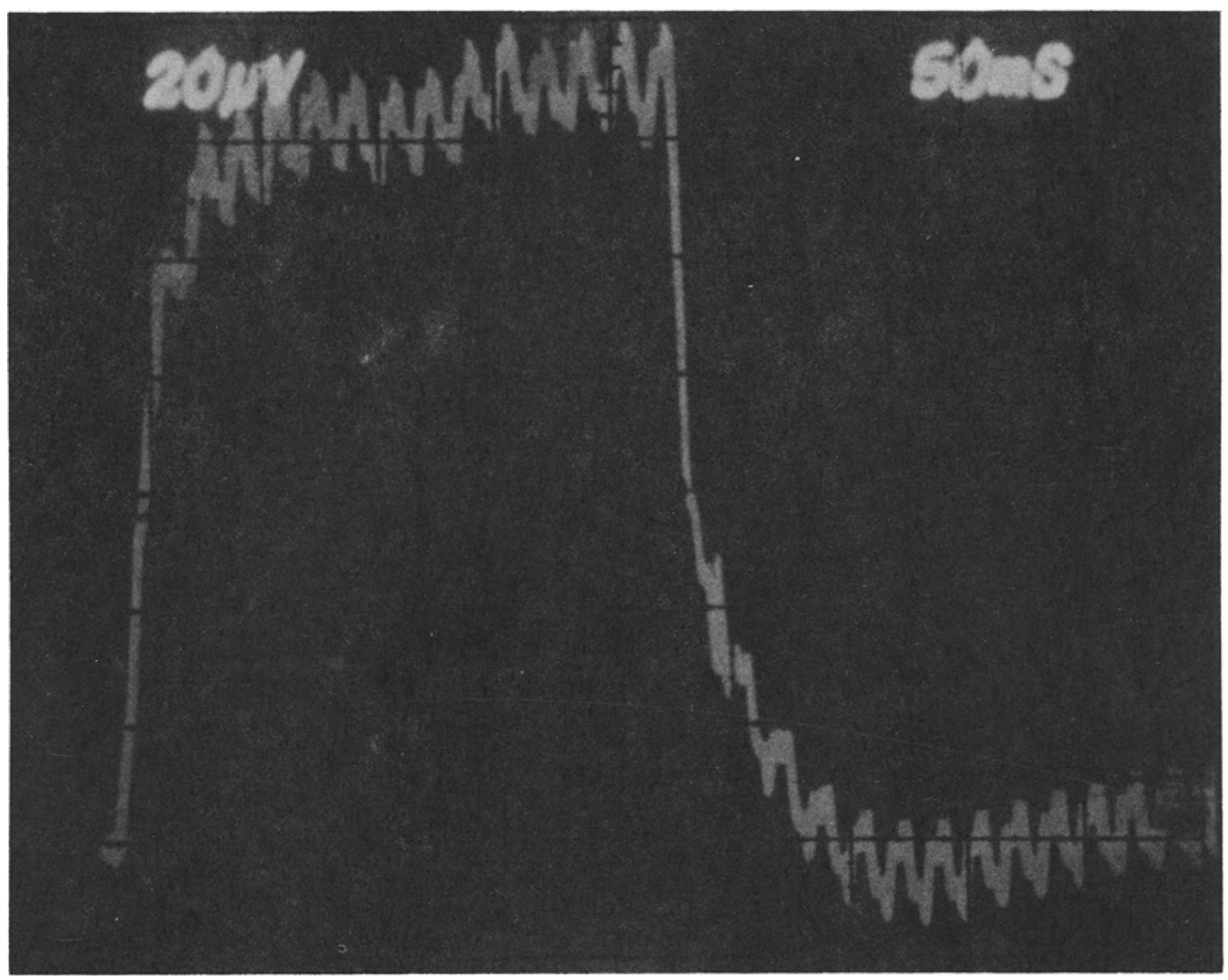

Figure 3. Pulse shape of stimulus line alone. Flow = 1.5 liters $/ \mathrm{min} ; 225-\mathrm{msec}$ duration.

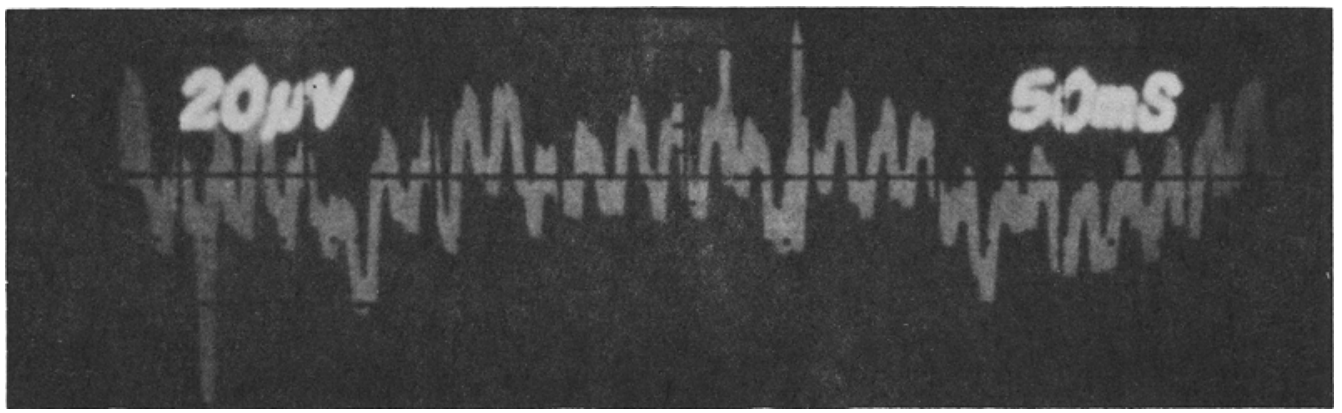

Figure 4. Oscillographic tracing of the output of the olfactometer at switching of pure and stimulus lines. Flow $=1.5$ liters $/ \mathrm{min}$ each; 225 -msec duration.

between the pure air line and the stimulus air line exists, a National Bureau of Standards traceable bubble flow meter (Hastings HBM-1) is used to match the outputs. To avoid auditory cues from the relay changes, white noise is administered to the subjects through earphones.

The pulse shape of the stimulus line alone was determined oscillographically by inserting a .001 -in.-diam iron-constantan thermocouple (Omega IRCO 001) just inside the output of the olfactometer. This pulse (Figure 3) was essentially a square wave. The flow rate was 1.5 liters $/ \mathrm{min}$ for $225 \mathrm{msec}$. To determine if there was a detectable differential flow between the two airstreams, even though they had been calibrated, the above procedure was repeated with both flows. Figure 4 shows no detectable excursion from the horizontal sweep at either onset or offset of the pulse during normal operation.

Since the calibration is not done on-line, Table 1 illustrates the range obtained using the $14 \times 2 \mathrm{~cm}$ flowover tube with a $0-$ to $10-\mathrm{ml} / \mathrm{min}$ EMFC. As can be seen, the stability of the instrument was quite good except at the extreme low end $(.3 \mathrm{ml} / 1.5$ liters $)$. The range producible by this instrument depends on the 
Table 1

Means (in Microvolts per Second), Standard Deviations, and Parts per Million of the Gas Chromatographic Analysis of the Olfactometer Output

\begin{tabular}{lcrrr}
\hline & $\mathrm{N}$ & Mean & SD & ppm \\
\hline $10 \mathrm{ml} / 1.5$ liters & 6 & 316,033 & 75.97 & 1.79 \\
$5 \mathrm{ml} / 1.5$ liters & 5 & 154,560 & 39.89 & .87 \\
$2.5 \mathrm{ml} / 1.5$ liters & 5 & 77,654 & 260.06 & .44 \\
$1.25 \mathrm{ml} / 1.5$ liters & 5 & 36,494 & 97.81 & .21 \\
$.62 \mathrm{ml} / 1.5$ liters & 5 & 16,724 & 37.35 & .09 \\
$.3 \mathrm{ml} / 1.5$ liters & 5 & 8,924 & 2466.5 & .05 \\
\hline
\end{tabular}

surface area of the flowover tube, the flow rate of the odor-bearing line, and the flow rate of the diluent airstream.

\section{APPLICATIONS}

This olfactometer with some modifications has been used to determine the flow thresholds of nonodorous air through the human nares at various temperature and humidity levels (Benignus \& Prah, 1980). This determi. nation was accomplished by interfacing a Békésy-type audiometer to an EMFC and allowing the subject to track his own threshold in a Békésy fashion. The current use is to evaluate the utility of the instrument in olfactory evoked potential research in which the generation of discrete stimuli and reliable timing of the stimulus pulses are required. In this regard, the $0-$ to $300-\mathrm{ml}$ EMFC was adjusted to a suprathreshold level for all subjects $(360 \mathrm{ppm})$.

\section{DISCUSSION}

The design of this olfactometer makes it an extremely flexible system for the generation and control of the stimulus, while permitting rapid modification of its function through the programming capabilities of the DBC. It is well adapted for evoked potential studies, threshold determinations, and sensory adaptation studies. In addition, the EMFCs, the timing precision of the $\mathrm{DBC}$, the use of nasal catheters, and the close control of the temperature permit accurate stimulus specification.

The olfactometer described is a significant improvement over previous designs in its use of a microcomputer to control the stimulus delivery parameters and the use of EMFCs to continuously regulate the airflows. The ability to maintain very close control permits assurance of constant airflow. This is especially important in the odorant air line, in which flow is a determinant of odorant level. EMFCs are more accurate and have more precise repeatability than do the more commonly used rotometers (Nelson, 1971). The EMFCs are controllable from external sources, allowing, if desired, the control of the flows to be set by the DBC for automated ascending and descending threshold determination.

Because of the use of velopharyngeal closure, the stimulus material does not go beyond the nasal cavities, thereby reducing the exposure risk of the subject to a minimal level.

Stimulus-correlated cues that may bias the subject have been minimized by calibrating the flow lines, maintaining airflow below threshold, and providing masking noise. Other cues and confounding variables have been avoided by thorough cleaning and the use of inert materials. Verification of contamination-free status by gas chromatography or some other analytical technique is essential.

All of these efforts are toward accurate stimulus specification. The techniques used here, including the use of nasal catheters and velopharyngeal closure, ensure maximum control over the stimulus. Free breathing or sniff methods neither control quantity of odorant that is actually inhaled by the subject nor assure that each subject receives the same stimulation unless a technique like that devised by Rehn (1978) and Teghtsoonian et al. (1978) is employed. This system will deliver to all subjects the same quantity of odorant material. Perhaps it is the lack of control over the stimulus that has retarded the explication of the olfactory system. This apparatus is a step closer to this goal.

\section{REFERENCES}

Air quality criteria for particulate matter (National Air Pollution Control Administration Publication No. AP-49). Washington, D.C: U.S. Government Printing Office, 1970.

Benignus, V. A., \& Prah, J. D. Flow thresholds of nonodorous air through the human naris as a function of temperature and humidity. Perception \& Psychophysics, 1980, 27, 569-573.

CAIN, W. S. Perception of odor intensity and the time-course of olfactory adaptation. American Society of Heating and Air Conditioning Engineers, 1974, 80, 153-175.

Davis, R. G. The microencapsulation of odorants as a method of stimulus control and delivery in studies of odor quality perception. Chemical Senses and Flavour, 1979, 4, 191-206.

DeVries, H., \& Stuiver, M. The absolute sensitivity of the human sense of smell. In W. A. Rosenblith (Ed.), Sensory communication. Cambridge, Mass: M.I.T. Press, 1961.

DravnieKs, A. Instrumental aspects of olfactometry. In D. G. Moulton, A. Turk, \& J. W. Johnston (Eds.), Methods in olfactory research. New York: Academic Press, 1975.

Foster, D., Scofield, E. H., \& Dallenbach, K. M. An olfactorium. American Journal of Psychology, 1950, 63, 430436.

Johnston, J. W. Quantification of olfactory stimuli. In C. C. Brown (Ed.), Methods in psychophysiology. Baltimore: William \& Wilkins, 1967.

LindVALL, T. On sensory evaluation of odorous air pollutant intensities. Nordisk Hygiensk Tidskrift (Supplementum 2), 1970, 1-181.

Mateson, J. F. Olfactometry: Its techniques and apparatus. Journal of the Air Pollution Control Association, 1955, 5. 167-170. 
Moze Ll, M. M. The chemical senses: II. Olfaction. In J. W. Kling \& L. A. Riggs (Eds.), Experimental psychology. New York: Holt, Rinehart, \& Winston, 1971.

NAdE R, J. S. An odor evaluation apparatus for field and laboratory use. American Industrial Hygiene Association, 1958, 19, 1-7.

Nelson, G. O. Controlled test atmospheres. Ann Arbor, Mich: Ann Arbor Science Publishers, 1971.

REHN, T. Perceived odor intensity as a function of air flow through the nose. Sensory Processes, 1978, 2, 198-205.

Schneider, R. A., \& Wolf, S. Olfactory perception thresholds for citral utilizing a new type olfactorium. Journal of Applied Physiology, 1955, 8, 337-342.

Stone, H., Pryor, G., \& Ste inmetz, G. The design and opera- tion of an improved olfactometer for behavioral and physiological investigation. Behavior Research Methods \& Instrumentation, 1969, 1, 153-156.

Teghtsoonian, R., Teghtsoonian, M., Berglund, B., \& BERgLUND, U. Invariance of odor strength with sniff vigor: An olfactory analogue to size and constancy. Journal of Experimental Psychology: Human Perception and Performance, 1978, 4, 144-152.

Weurman, C. Sampling in airborne odorant analysis. In A. Turk, J. W. Johnston, \& D. G. Moulton (Eds.), Human responses to environmental odors. New York: Academic Press, 1974.

(Received for publication June 16, 1980; revision accepted October $13,1980$. ) 